\title{
Decrease in lipid levels of syncytiotrophoblast micro-particles reduced their potential to inhibit endothelial cell proliferation
}

\author{
Anurag Kumar Gupta • Wolfgang Holzgreve • \\ Sinuhe Hahn
}

Received: 18 January 2007 / Accepted: 5 July 2007 / Published online: 25 July 2007

(C) Springer-Verlag 2007

\begin{abstract}
Background Preeclampsia is characterized by damage to the maternal endothelium that has been suggested to be mediated in part by elevated shedding of inflammatory placental syncytiotrophoblast micro-particles (STBM) into the maternal circulation. Previously, we have shown that STBM, prepared by three different methods: mechanical dissection, in vitro placental explants culture and perfusion of placenta, can inhibit endothelial cell proliferation. Only mechanically prepared STBM induced apoptosis in the endothelial cells. Now, we have examined lipid levels in the three STBM preparations and their differential responses on endothelial cells.

Methods We examined the lipid levels in the three STBM preparations using thin layer chromatography. Furthermore, the effects of reduced lipid levels in the three STBM preparations using the pharmacological agent methyl- $\beta$ cyclodextrin were examined on endothelial cell proliferation and apoptosis.

Results Among the three STBM preparations, mechanical STBM contained highest levels of lipids. The reduction in lipid levels in mechanical STBM reduced their potential to inhibit human umbilical vein endothelial cells (HUVEC) proliferation and blocked their potential to induce apoptosis. No similar effect was observed following lipid reduction in the two other STBM preparations.

Conclusions As it has been suggested that mechanically derived STBM may more closely resemble placental microparticles generated in preeclampsia, our data suggest that
\end{abstract}

A. K. Gupta $(\bowtie) \cdot$ W. Holzgreve · S. Hahn

Laboratory for Prenatal Medicine,

University Women's Hospital/Department of Research,

University Hospital, Hebelstrasse, 20, 4031 Basel, Switzerland

e-mail: gkumar@uhbs.ch lipid content may play a role in the anti-endothelial defects present in this disease.

Keywords Preeclampsia $\cdot \mathrm{STBM} \cdot \mathrm{HUVEC}$. Methyl- $\beta$-cyclodextrin · Lipids
Abbreviations
PBS Phosphate buffered saline
STBM Syncytiotrophoblast micro-particles
$\mathrm{M} \beta \mathrm{CD} \quad$ Methyl- $\beta$-cyclodextrin
HUVEC Human umbilical vein endothelial cells

\section{Introduction}

Preeclampsia is a severe obstetric disorder affecting about $2-7 \%$ of pregnant women $[1,2]$. Its features include high blood pressure, proteinuria and edema [1]. This disorder is associated with a considerable risk for feto-maternal morbidity and mortality [3]. Recent research has indicated that these diverse arrays of symptoms may be the result of an excessive maternal systemic inflammatory response to pregnancy, involving activation of the innate immune system and damage of the maternal endothelial compartment $[3,4]$.

It is well established that the placenta plays a central role in the underlying etiology of preeclampsia and it has been suggested that the elevated release of syncytiotrophoblast derived micro-particles (STBM) may contribute to this pathology, which affects both the maternal endovasculature and immune systems $[2,4,5]$. In normal pregnancy, trophoblast cells and STBM are spontaneously released from the placenta as a sign of normal placental physiologic turnover [6-8]. This phenomenon has been associated with the mild inflammatory state accompanying pregnancy [3, 9]. 
This proposal is supported by the observations that STBM are shed in greater quantities by the placenta in preeclampsia and that STBM generated in vitro by mechanical dissection of the placenta suppresses endothelial cell proliferation [10]. This increased shedding of sub-cellular material parallels the raise in circulating cell-free fetal DNA [11, 12], demonstrating the increased placental dysfunction associated with the disorder [13]. Therefore, it has been proposed that the increased shedding of STBM into the maternal blood of women with preeclampsia could be responsible for the manifest dysfunction of the vascular endothelium [9].

In order to study the effect of STBM in greater detail, recently we have characterized three different STBM preparations [14]. The effects of three STBM preparations were examined on endothelial cells and purified T cells [14, 15]. Among the three STBM preparations, mechanically derived STBM (mSTBM) preparation largely behaved in a different manner than the other two preparations, i.e. villous explants STBM (vSTBM) and placental perfusion STBM (pSTBM) [14]. We observed that only mSTBM significantly inhibited endothelial cell proliferation and induced apoptosis of these cells [14].

It has been proposed that preeclampsia and atherosclerosis share similar symptoms [16-18]. In this regard lipids have been shown to play an important role in the pathogenesis of both diseases [16-18]. As STBM are the products of syncytiotrophoblast cell layer of the villous tree, they should contain membrane lipids, which might play a role in the pathogenesis of preeclampsia. Therefore, in this study, we examined lipids levels in different STBM preparations. Moreover, lipid levels could be reduced from the STBM preparations and the effects of these various STBM preparations were examined on endothelial cell proliferation and apoptosis.

\section{Materials and methods}

Preparation of syncytiotrophoblast micro-particles

This study was approved by the Cantonal Institutional Review Board of Basel, Switzerland. Written informed consent was requested in all instances. Placentae and umbilical cords were obtained after normal term delivery or elective cesarean section from uncomplicated pregnancies and processed within 30-90 min of collection. STBM were prepared by three different methods as described previously [14]. The protein content in each STBM preparation was quantified with the advanced protein assay reagent (Cytoskeleton Inc. Denver, CO, USA) according to the manufacturer's instructions.

\section{Lipid analysis of STBM}

One hundred micrograms of the each STBM preparation was extracted with chloroform. After centrifugation for
$1 \mathrm{~min}$ the lipid containing lower organic layer was collected and applied to the pre-coated thin layer chromatography (TLC) plate. TLC was carried out in the pre-saturated chamber. The running buffer consisted of chloroform:methanol:water:ammonium hydroxide 65:30:3:2 (v/v/v/v). The TLC run was monitored until the top of the plate. The separated lipids were visualized by staining with mosatin stain [4\% ammonium molybdate tetrahydrate $(\mathrm{w} / \mathrm{v})+0.08 \%$ cesium sulfate tetrahydrate $\left.(\mathrm{w} / \mathrm{v})+30 \% \mathrm{H}_{2} \mathrm{SO}_{4}(10 \%, \mathrm{v} / \mathrm{v})\right]$ and charring at $200^{\circ} \mathrm{C}$.

\section{Lipid reduction from STBM}

One hundred micrograms of the each STBM preparation was incubated with varying concentrations (2-8\%) of methyl- $\beta$-cyclodextrin $(\mathrm{M} \beta \mathrm{CD})$ at $\mathrm{RT}$ for $30 \mathrm{~min}$. After incubation with $\mathrm{M} \beta \mathrm{CD}$ STBM were pelleted down and resuspended in sterile phosphate buffered saline (PBS). Lipids were extracted from $\mathrm{M} \beta \mathrm{CD}$ treated STBM and the supernatants as described above. TLC was carried out to confirm the lipid reduction from the STBM preparations and their appearance in the supernatants.

\section{Cell proliferation assay}

Human umbilical vein endothelial cells were isolated from normal term human umbilical cord by the method described by Jaffe et al. [19, 20] and stored in liquid nitrogen until use. Cells from passage 2 to 7 were used for all experiments. HUVEC proliferation was measured using cell proliferation kit (Roche Diagnostics, Basel, Switzerland), which measures BrdU incorporation during DNA synthesis. In brief, $1.5 \times 10^{4}$ cells were plated onto $1 \%$ gelatin coated 96 well microtiter plates (Nunc, Denmark) in endothelial serum-free medium (E-SFM) supplemented with $20 \mathrm{ng} / \mathrm{ml}$ human recombinant basic fibroblast growth factor (bFGF) and $10 \mathrm{ng} / \mathrm{ml}$ human recombinant epidermal growth factor (EGF) (GIBCO Invitrogen Life Technologies, Grand Island, NY, USA). After $6 \mathrm{~h}$, the culture medium was replaced with untreated STBM or $\mathrm{M} \beta \mathrm{CD}$ treated STBM diluted in DMEM:F12 medium supplemented with $1 \%$ antimycotic/antibiotics, $10 \% \mathrm{FCS}, 50 \mathrm{U} / \mathrm{ml}$ aprotinin, $25 \mathrm{U} /$ ml heparin (DMEM:F12 complete medium). The cells were then incubated for $22 \mathrm{~h}$, followed by an additional incubation with $10 \mu \mathrm{M}$ of BrdU for a further $18 \mathrm{~h}$. The amount of incorporated BrdU was detected using an ELISA reader (Molecular Devices, Sunnyvale, CA, USA) according to the manufacturer's instructions.

Human umbilical vein endothelial cells apoptosis

Apoptosis was measured using a commercial cellular DNA fragmentation ELISA kit (Roche Diagnostics, Germany). 
This assay measures the release of BrdU labeled DNA fragments into the cytoplasm during apoptosis. In brief, $2 \times 10^{4}$ HUVEC were plated in 96 well microtiter plates (Nunc, Denmark) in E-SFM culture medium supplemented with $20 \mathrm{ng} / \mathrm{ml} \mathrm{bFGF}$ and $10 \mathrm{ng} / \mathrm{ml}$ EGF. After $6 \mathrm{~h} 10 \mu \mathrm{M}$ BrdU was added and incubated for a further $18 \mathrm{~h}$. Following BrdU labeling, the cell monolayer was washed with sterile PBS, then $\mathrm{M} \beta \mathrm{CD}$ treated and untreated STBM containing DMEM:F12 complete medium was added for $24 \mathrm{~h}$. In positive control wells, apoptosis was induced with $5 \mu \mathrm{M}$ staurosporine (Sigma Chemicals, St Louis, MO, USA), a potent inducer of apoptosis, for $3 \mathrm{~h}$. The detection of BrdU labeled apoptotic DNA fragments was performed according to the manufacturer's instructions.

\section{Results}

Mechanical STBM were rich in lipids

All three STBM preparations were applied to TLC plate after the lipid extraction as described in "Materials and methods". Five spots were observed on the run front of the TLC plate. Using commercially available cholesterol as a standard, spot at the top of the TLC plate run was identified as cholesterol. This analysis also revealed that the lipid concentration in the mSTBM is greater than the other two STBM preparations (Fig. 1). These data indicate the possibility of mSTBM to be richer in membrane materials than the other two STBM preparations at equal protein concentration.

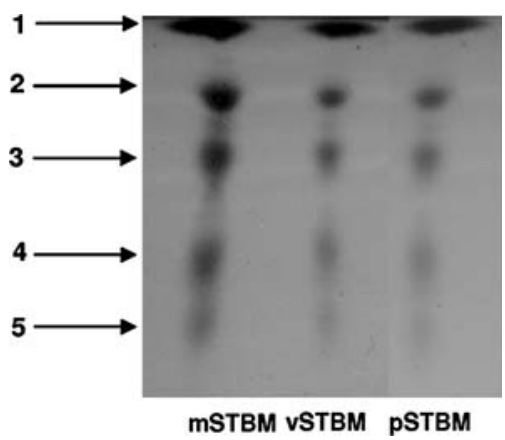

Fig. 1 Thin layer chromatographic analysis of different STBM preparations. Lipids were extracted from $100 \mu \mathrm{g}$ of the each STBM preparation as described in "Materials and methods" and applied to the precoated TLC plate. TLC was carried out in the pre-saturated chamber. The TLC run was monitored until the top of the plate. The separated lipids were visualized after staining. This analysis revealed five lipid spots in all STBM preparations. This analysis also confirmed that the $m S T B M$ are rich in membrane lipids
Methyl- $\beta$-cyclodextrin efficiently reduced lipids from STBM

It has been previously shown that membrane lipids can efficiently be depleted using M $\beta C D$ [21]. Therefore, we reduced lipids from all the three STBM preparations as described in "Materials and methods". In a dose-dependent manner large amounts of lipids could be reduced from mSTBM (Fig. 2a). Moreover, the reduced lipids appeared in the supernatant of the treated mSTBM (Fig. 2a). On the other hand, low levels of lipids present in pSTBM and vSTBM could be depleted by the lowest concentration of $\mathrm{M} \beta \mathrm{CD}$ (2\%) (Fig. 2b). These observations suggest that $\mathrm{M} \beta \mathrm{CD}$ could effectively reduce membrane lipids in all three STBM preparations.

Lipid reduction from mechanically derived STBM reduced their potential to inhibit HUVEC proliferation

It has been previously reported that the three STBM preparations can inhibit endothelial cell proliferation in a dose dependant manner [14]. Therefore, we examined whether the lipid depletion of the three different STBM affected HUVEC proliferation. The effects of equal amounts of normal and lipid depleted STBM, as determined by protein content, were compared on HUVEC proliferation. For this analysis we made use of highest concentration $(12 \mu \mathrm{g} / \mathrm{well})$ of each STBM preparation as this dose has previously been shown to inhibit maximal HUVEC proliferation [14]. We observed that upon lipid reduction the inhibition of HUVEC proliferation by mSTBM $(90 \%$ at $12 \mu \mathrm{g}$ untreated
A

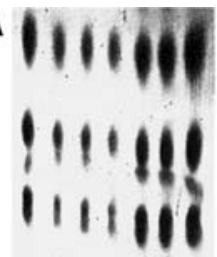

1223445667

Lane 1: Untreated mSTBM

Lane 2: Treated $\mathrm{mSTBM}$ with $2 \% \mathrm{M} \beta \mathrm{CD}$

Lane 3: Treated mSTBM with $4 \% \mathrm{MBCD}$ Lane 4: Treated $\mathrm{mSTBM}$ with $8 \% \mathrm{MBCD}$ Lane 5: supernatant after $2 \% \mathrm{M} \beta \mathrm{CD}$ treatment Lane 6: supernatant after $4 \% \mathrm{MBCD}$ treatment Lane 7: supernatant after $8 \% \mathrm{M} \beta C D$ treatment

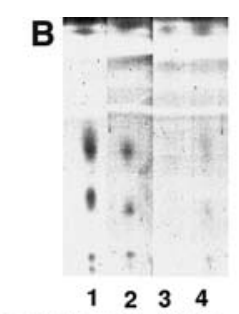
Lane 2: Untreated pSTBM Lane 3: Treated vSTBM with $2 \% \mathrm{MBCD}$ Lane 4: Treated pSTBM with $2 \% \mathrm{MBCD}$
Lane 1: Untreated vSTBM

Fig. 2 Lipid reduction in different STBM preparations. One hundred micrograms of the each STBM preparation was incubated with varying doses of $\mathrm{M} \beta \mathrm{CD}$ for $30 \mathrm{~min}$ and then lipids were extracted as described in "Materials and methods". Lipids were also extracted from the supernatants of $\mathrm{M} \beta \mathrm{CD}$ treated STBM. Treated and untreated STBM were applied to the pre-coated TLC plate. TLC was carried out in the presaturated chamber. This analysis revealed that lipids from $m S T B M$ could efficiently be reduced in the dose-dependant manner and depleted lipids could be observed in the supernatant (a). On the other hand, lipids from $p S T B M$ and $\nu S T B M$ could be depleted totally with the lowest concentration ( $2 \%)$ of $\mathrm{M} \beta \mathrm{CD}(\mathbf{b})$ 
mSTBM per well) was significantly reduced (58\% at $12 \mu \mathrm{g}$ $\mathrm{M} \beta \mathrm{CD}$ treated $\mathrm{mSTBM}$ per well). On the other hand, lipid depletion of the villous explants STBM and placental perfusion STBM, at similar dose, did not lead to a decrease in the inhibition of endothelial cell proliferation (Fig. 3).

Lipid reduction from mechanically derived STBM abolished their potential to induce HUVEC apoptosis

We have previously reported that among the three STBM preparations only mSTBM induced HUVEC apoptosis in a dose-dependant manner [14]. Therefore, we examined whether lipid depletion of mSTBM reduced their potential to induce apoptosis in HUVEC. We observed that the apoptosis induced by $\mathrm{mSTBM}$ at highest concentration, $28 \%$ apoptosis at $12 \mu \mathrm{g}$ untreated mSTBM per well, could be abolished after lipid reduction in this STBM preparation when used at similar dose (Fig. 4).

\section{Discussion}

In this study, we extend upon previous study in which three different STBM preparations were characterized morphologically and functionally [14]. Now, we have examined lipid levels in these STBM preparations using TLC. In this regard it has been previously reported that high lipid content leads to endothelial cell dysfunction in many diseases such as hypercholesterolemia, preeclampsia, inflammation and hypertension $[18,22]$. Our TLC analysis of the three STBM preparations revealed $\mathrm{mSTBM}$ has the highest lipids

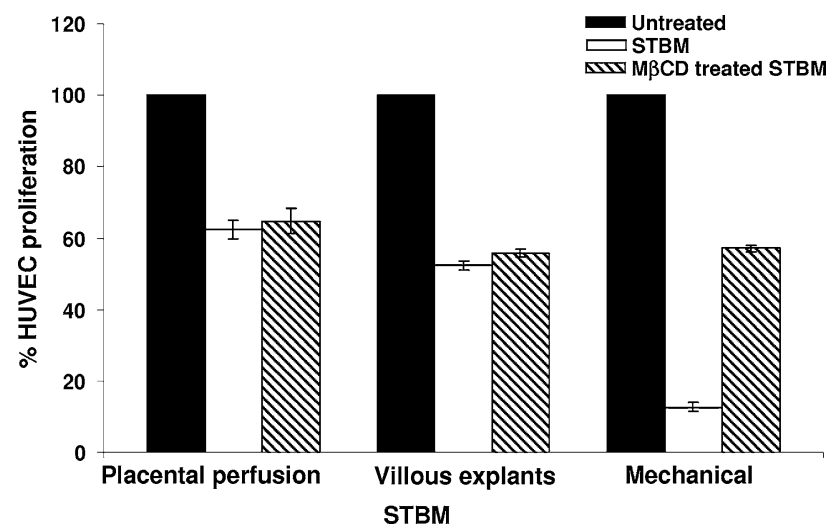

Fig. 3 Reduction in the inhibition of endothelial cell proliferation by mSTBM. Sub-confluent HUVEC cells were incubated for $40 \mathrm{~h}$ with each STBM preparation (untreated or M $\beta$ CD treated) in DMEM:F12 complete medium. Cell proliferation was measured as described in "Materials and methods" and is represented as the percentage of cell proliferation relative to untreated control as $100 \%$ cell proliferation. This data suggested that the HUVEC proliferation inhibition could be reduced only by lipid-depleted $m S T B M$ not by the other two STBM preparations. Analysis of STBM preparations from six placentae is illustrated. Error bars represent standard deviation $( \pm \mathrm{SD})$

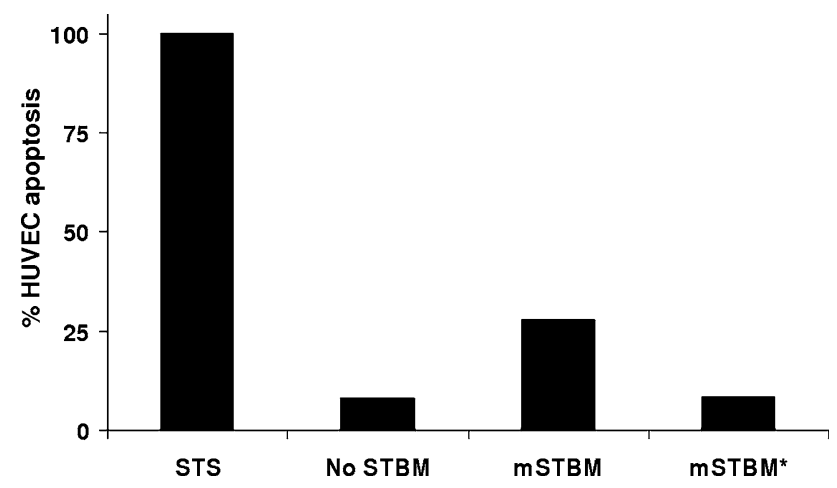

Fig. 4 Inhibition of endothelial cell apoptosis by mSTBM. Confluent HUVEC were incubated with $12 \mu \mathrm{g} /$ well concentration of untreated and $\mathrm{M} \beta \mathrm{CD}$ treated $m S T B M\left(m S T B M^{*}\right)$ preparations and apoptosis was measured as described in "Materials and method". Five micromolar staurosporine (STS) was used as positive control. Apoptosis is expressed as percentage relative to staurosporine-induced apoptosis, which was designated as $100 \%$. A single representative experiment is shown out of three experiments

content, when compared to the other two STBM preparations. As STBM are derived from the syncytiotrophoblast membrane layer of the placenta, it is most likely that the lipids present in these STBM preparations are of membrane origin. Large amounts of lipids present in MSTBM indicate the high levels of membranous material present in this preparation, which is in agreement with our previous observation where mSTBM contains large amounts of GPI, anchored lipid membrane enzyme, placental like alkaline phosphatase (PLAP) [14].

Three STBM preparations have been shown to reduce HUVEC proliferation, among them MSTBM had the greatest effect and induced endothelial cell death by apoptosis [14]. As this functional disparity of the STBM on endothelial cell culture was not clear at that time, we examined whether this effect is due to the excess lipid content present in mSTBM. For this purpose we reduced lipids from the three STBM preparations using $\mathrm{M} \beta \mathrm{CD}$. We observed that lipid decrease in the STBM preparations resulted in the reduced inhibition of HUVEC proliferation by mSTBM. No similar effects were noticed with the other two STBM preparations. Moreover, lipid reduction in $\mathrm{MSTBM}$ blocked their potential to induce apoptosis. Hence, these observations provide further evidence that the three STBM differ in their biochemical composition as well. As the extent of HUVEC proliferation inhibition by lipid-depleted mSTBM was similar to that mediated by PSTBM or VSTBM in their native form, suggesting that lipids contribute partially to the inhibition of HUVEC proliferation and subsequent apoptosis. Therefore, these data suggest that excessive HUVEC proliferation inhibition and apoptosis induction mediated by the mSTBM in its native form may be due to the high levels of lipids present in these particles. 
It has been suggested that in vivo the shedding of placental particles is a continuous feature of normal pregnancy, which increases during pregnancy due to an increase in placental size. In this context, it has been proposed the vSTBM and pSTBM prepared from explants cultures or placental perfusion, respectively, may more closely mimic micro-particles released by normal syncytiotrophoblast turnover [23]. On the other hand mSTBM, which are generated by mechanical disruption of villous integrity, may be more similar to particles released by the process of aponecrosis, which have been proposed to occur in the preeclamptic placentae due to oxidative stress [23]. Therefore, it will be interesting to examine in future whether STBM from preeclamptic patients exhibit higher levels of lipids akin to what we have observed in mSTBM.

Acknowledgments We thank Dr. Sachin Shelke for his kind help in the TLC of STBM. This work was supported in part by a grant from The Special Non-Invasive Advances in Fetal and Neonatal Evaluation Network (SAFE) (No. DMS-2007).

\section{References}

1. Redman CW, Sargent IL (2005) Latest advances in understanding preeclampsia. Science 308:1592-1594

2. Roberts JM, Cooper DW (2001) Pathogenesis and genetics of preeclampsia. Lancet 357:53-56

3. Redman CW, Sargent IL (2004) Preeclampsia and the systemic inflammatory response. Semin Nephrol 24:565-570

4. Redman CW, Sargent IL (2003) Pre-eclampsia, the placenta and the maternal systemic inflammatory response-a review. Placenta 24(Suppl A):S21-S27

5. Redman CW, Sargent IL (2000) Placental debris, oxidative stress and pre-eclampsia. Placenta 21:597-602

6. Chua S, Wilkins T, Sargent I, Redman C (1991) Trophoblast deportation in pre-eclamptic pregnancy. Br J Obstet Gynaecol 98:973-979

7. Huppertz B, Frank HG, Kingdom JC, Reister F, Kaufmann P (1998) Villous cytotrophoblast regulation of the syncytial apoptotic cascade in the human placenta. Histochem Cell Biol 110:495-508

8. Johansen M, Redman CW, Wilkins T, Sargent IL (1999) Trophoblast deportation in human pregnancy-its relevance for preeclampsia. Placenta 20:531-539

9. Borzychowski AM, Sargent IL, Redman CW (2006) Inflammation and pre-eclampsia. Semin Fetal Neonatal Med 11:309-316
10. Knight M, Redman CW, Linton EA, Sargent IL (1998) Shedding of syncytiotrophoblast microvilli into the maternal circulation in pre-eclamptic pregnancies. Br J Obstet Gynaecol 105:632-640

11. Lo YM, Leung TN, Tein MS, Sargent IL, Zhang J, Lau TK, Haines CJ, Redman CW (1999) Quantitative abnormalities of fetal DNA in maternal serum in preeclampsia. Clin Chem 45:184-188

12. Zhong XY, Laivuori H, Livingston JC, Ylikorkala O, Sibai BM, Holzgreve W, Hahn S (2001) Elevation of both maternal and fetal extracellular circulating deoxyribonucleic acid concentrations in the plasma of pregnant women with preeclampsia. Am J Obstet Gynecol 184:414-419

13. Hahn S, Holzgreve W (2002) Fetal cells and cell-free fetal DNA in maternal blood: new insights into pre-eclampsia. Hum Reprod Update 8:501-508

14. Gupta AK, Rusterholz C, Huppertz B, Malek A, Schneider H, Holzgreve W, Hahn S (2005) A comparative study of the effect of three different syncytiotrophoblast micro-particles preparations on endothelial cells. Placenta 26:59-66

15. Gupta AK, Rusterholz C, Holzgreve W, Hahn S (2005) Syncytiotrophoblast micro-particles do not induce apoptosis in peripheral $\mathrm{T}$ lymphocytes, but differ in their activity depending on the mode of preparation. J Reprod Immunol 68:15-26

16. Var A, Kuscu NK, Koyuncu F, Uyanik BS, Onur E, Yildirim Y, Oruc S (2003) Atherogenic profile in preeclampsia. Arch Gynecol Obstet 268:45-47

17. Bayhan G, Kocyigit Y, Atamer A, Atamer Y, Akkus Z (2005) Potential atherogenic roles of lipids, lipoprotein(a) and lipid peroxidation in preeclampsia. Gynecol Endocrinol 21:1-6

18. Belo L, Caslake M, Gaffney D, Santos-Silva A, Pereira-Leite L, Quintanilha A, Rebelo I (2002) Changes in LDL size and HDL concentration in normal and preeclamptic pregnancies. Atherosclerosis $162: 425-432$

19. Jaffe EA, Nachman RL, Becker CG, Minick CR (1973) Culture of human endothelial cells derived from umbilical veins. Identification by morphologic and immunologic criteria. J Clin Invest 52:2745-2756

20. Nachman RL, Jaffe EA (2004) Endothelial cell culture: beginnings of modern vascular biology. J Clin Invest 114:1037-1040

21. Hao M, Mukherjee S, Maxfield FR (2001) Cholesterol depletion induces large scale domain segregation in living cell membranes. Proc Natl Acad Sci USA 98:13072-13077

22. Thomas JP, Geiger PG, Girotti AW (1993) Lethal damage to endothelial cells by oxidized low density lipoprotein: role of selenoperoxidases in cytoprotection against lipid hydroperoxideand iron-mediated reactions. J Lipid Res 34:479-490

23. Huppertz B, Kingdom J, Caniggia I, Desoye G, Black S, Korr H, Kaufmann P (2003) Hypoxia favours necrotic versus apoptotic shedding of placental syncytiotrophoblast into the maternal circulation. Placenta 24:181-190 